\title{
Multiple treatment modalities for brain metastasis in patients with EGFR-mutant non-small-cell lung cancer
}

This article was published in the following Dove Press journal:

OncoTargets and Therapy

\author{
Haiyang Wangl,* \\ Xiaoqing $\mathrm{Yu}^{\mathrm{l}, *}$ \\ Yun Fan ${ }^{2}$ \\ Youhua Jiang ${ }^{2}$ \\ 'Department of Second Clinical \\ Medical College, Zhejiang Chinese \\ Medical University, Hangzhou, \\ Zhejiang, People's Republic of China; \\ ${ }^{2}$ Key Laboratory of Diagnosis and \\ Treatment Technology on Thoracic \\ Oncology (Esophagus, Lung), Zhejiang \\ Cancer Hospital, Hangzhou, Zhejiang, \\ People's Republic of China
}

*These authors contributed equally to this work
Correspondence: Youhua Jiang Key Laboratory of Diagnosis and Treatment Technology on Thoracic Oncology (Esophagus, Lung), Zhejiang Cancer Hospital, No.I East Banshan Road, Gongshu District, Hangzhou 310022, Zhejiang, People's Republic of China

Tel $+8657|8812219|$

Fax +8657188122589

Email 461426358@qq.com
Background: There are many controversies concerning the best management of epidermal growth factor receptor (EGFR)-mutant non-small-cell lung cancer (NSCLC) patients with brain metastases (BMs). The use of upfront EGFR tyrosine kinase inhibitors (TKIs) and the withholding of local therapies or upfront radiation therapies (RTs) remain controversial. Available treatment options include local therapies such as whole-brain radiation therapy (WBRT), stereotactic radiosurgery (SRS) and surgery, EGFR-TKIs, and chemotherapy. However, the optimal management of combination therapies is still under consideration.

Patients and methods: A total of 45 EGFR-mutated NSCLC patients with BMs were included. All patients successively received EGFR-TKIs, RT (WBRT or SRS), and chemotherapy between 2010 and 2015 at Zhejiang Cancer Hospital. Patient follow-up was conducted by telephone until February 2017. The treatment response was evaluated, and survival data were collected and analyzed by Kaplan-Meier analysis and the Cox regression method.

Results: The median overall survival (OS) was 28 months. Patients with the exon 19 deletion showed the strongest trend toward a longer median OS compared to patients with the exon 21 L858R mutation (not reached vs 26.5 months, $P=0.0969$ ). There was no difference in OS between the upfront RT group and the deferral group ( 26.5 vs 28 months, $P=0.57$ ), and similar results were found between the first-line chemotherapy group and the EGFR-TKI group (28 vs 23.2 months, $P=0.499)$. In multivariate analysis, the prognosis correlated with EGFR mutation type $(P=0.017)$.

Conclusion: EGFR-mutant NSCLC patients with BM benefited from the combination and sequential therapies of EGFR-TKIs, chemotherapy, and RTs. Patients with the EGFR exon 19 deletion may have a better OS. However, the optimal timing of RT interval remains to be explored.

Keywords: epidermal growth factor receptor, tyrosine kinase inhibitors, brain metastases, non-small-cell lung cancer, pemetrexed, whole-brain radiation therapy

\section{Introduction}

Brain metastases (BMs) are a common cause of morbidity and mortality in patients with non-small-cell lung cancer (NSCLC), and BMs develop in $\sim 25 \%-40 \%$ of patients with advanced adenocarcinomas; moreover, the incidence of BMs is still increasing. ${ }^{1,2}$ Patients with epidermal growth factor receptor (EGFR)-mutant NSCLC may have a higher likelihood of being diagnosed with BMs because of prolonged survival from targeted systemic agents and the increased quality of central nervous system imaging. ${ }^{3}$ The median overall survival (OS) of an unselected population of EGFR-mutant and non-EGFR-mutant NSCLC patients with BMs reportedly ranged 
from 3 to 15 months, ${ }^{4}$ whereas the median OS after BMs of 19-58 months in patients with EGFR-mutant NSCLC was observed. ${ }^{5,6}$ Historically, therapeutic options for BMs have been limited to local therapies such as whole-brain radiation therapy (WBRT), stereotactic radiosurgery (SRS), surgery, or a combination of the above. Due to concerns about inadequate central nervous system penetration, chemotherapy is not typically a standard primary treatment for BMs. ${ }^{7}$ However, previously published studies describing the use of combined cisplatin and pemetrexed therapy confirmed good tolerability and efficiency in managing NSCLC patients with inoperable BMs. ${ }^{8,9}$ During the last decade, EGFR-tyrosine kinase inhibitors (TKIs) have been successfully employed in NSCLC patients based on the identification of EGFR gene mutations; however, EGFR-TKIs have also been demonstrated to be a potential treatment of choice for BMs from NSCLC patients harboring an activating EGFR mutation. ${ }^{10-16}$ In addition, some studies showed that the combination of RT and EGFR-TKIs produced superior outcomes for patients with EGFR mutations and BMs. 5,6,17,18

There are still several controversies concerning the management of EGFR-mutant NSCLC patients with BMs. The use of upfront EGFR-TKIs and the withholding of local therapies or upfront radiation therapies (RTs) remain controversial. Available treatment options include local therapies such as WBRT, SRS and surgery, EGFR-TKIs, and chemotherapy. To evaluate the efficacy of EGFR-mutant NSCLC patients with BM receiving multiple regimens and to analyze the prognostic factors, we retrospectively investigated 45 patients with EGFR-mutant NSCLC who developed BM between 2010 and 2015 and were successively treated with EGFR-TKIs, pemetrexed-based chemotherapy and radiotherapy.

\section{Patients and methods}

\section{Patients}

In this study, we retrospectively enrolled and analyzed 45 EGFR-mutated NSCLC patients with BMs who systematically received EGFR-TKIs (icotinib, gefitinib, erlotinib, or osimertinib), pemetrexed-based chemotherapy, and local therapies (WBRT or SRS) between 2010 and 2015 at Zhejiang Cancer Hospital. All patients were histologically diagnosed with NSCLC, and EGFR mutations were detected by the amplification refractory mutation system analysis, which identifies tumor lesions with EGFR mutations. BM in these patients was confirmed by magnetic resonance imaging. All patients completed clinical and follow-up evaluations (Table 1). The research was approved by the ethical committee of Zhejiang Cancer Hospital, including verbal informed consent being obtained from all participants. We confirm that patient data confidentiality was maintained.

Table I Patient characteristics at baseline

\begin{tabular}{|c|c|c|}
\hline Characteristics & $\mathbf{N}$ & $\%$ \\
\hline \multicolumn{3}{|l|}{ Gender } \\
\hline Male & 20 & 44.4 \\
\hline Female & 25 & 54.5 \\
\hline \multicolumn{3}{|l|}{ Age, years } \\
\hline$<65$ & 37 & 82.2 \\
\hline$\geq 65$ & 8 & 17.8 \\
\hline \multicolumn{3}{|l|}{ Smoking } \\
\hline Never & 29 & 64.4 \\
\hline Slight & 5 & 11.1 \\
\hline Heavy & 11 & 24.4 \\
\hline \multicolumn{3}{|l|}{ KPS } \\
\hline$<90$ & 38 & 84.4 \\
\hline$\geq 90$ & 7 & 15.6 \\
\hline \multicolumn{3}{|l|}{ RTOG GPA } \\
\hline $0-2$ & 27 & 60.0 \\
\hline $2.5-4$ & 18 & 40.0 \\
\hline \multicolumn{3}{|l|}{ Extracranial metastasis } \\
\hline No & 18 & 40.0 \\
\hline Yes & 27 & 60.0 \\
\hline \multicolumn{3}{|l|}{ No. of intracranial metastases } \\
\hline I & 14 & 31.1 \\
\hline 2 & 5 & II.I \\
\hline$>2$ & 26 & 57.8 \\
\hline \multicolumn{3}{|l|}{ Symptom when diagnosis } \\
\hline Without & 37 & 82.2 \\
\hline With & 8 & 17.8 \\
\hline \multicolumn{3}{|l|}{ Histology } \\
\hline Adenocarcinoma & 41 & 91.1 \\
\hline Others & 4 & 8.9 \\
\hline \multicolumn{3}{|l|}{ Mutation } \\
\hline Exon 19 deletion mutation & 22 & 48.9 \\
\hline Exon 2I L858R mutation & 23 & 51.1 \\
\hline \multicolumn{3}{|l|}{ First-line treatment } \\
\hline Chemotherapy & 28 & 62.2 \\
\hline EGFR-TKIs & 17 & 37.8 \\
\hline \multicolumn{3}{|l|}{ Chemotherapy } \\
\hline Dual agents & 30 & 66.7 \\
\hline Single agent & 15 & 33.3 \\
\hline \multicolumn{3}{|l|}{ Therapy for BM } \\
\hline Ist line & 19 & 42.2 \\
\hline $2 n d$ or 3rd line & 26 & 57.8 \\
\hline \multicolumn{3}{|l|}{ RT } \\
\hline WBRT & 38 & 84.8 \\
\hline SRS & 5 & 11.4 \\
\hline Combination & 1 & 2.3 \\
\hline \multicolumn{3}{|c|}{ Interval between RT and diagnosis } \\
\hline$\leq 3$ months & 30 & 66.7 \\
\hline$>3$ months & 15 & 33.3 \\
\hline
\end{tabular}

Abbreviations: BM, brain metastasis; EGFR-TKI, epidermal growth factor receptortyrosine kinase inhibitor; GPA, graded prognostic assessment; KPS, Karnofsky Performance Scale; RT, radiation therapies; RTOG, radiation therapy oncology group; SRS, stereotactic radiosurgery; WBRT, whole-brain radiation therapy. 


\section{Evaluation of treatment responses and toxicity}

The objective tumor response was assessed according to the Response Evaluation Criteria in Solid Tumors version 1.1, which was divided into complete response (CR), partial response (PR), stable disease (SD), and progressive disease (PD). Adverse reactions were evaluated based on the CTCAE 3.0 edition (Common Terminology Criteria for Adverse Events version 3.0) every month.

\section{Study design}

Using patient hospital records, follow-up registration records, and follow-up phone records, we collected clinical and survival information for the patients. The following data were collected and recorded: age, sex, smoking status, Karnofsky Performance Scale (KPS) at the time of BM, number of BMs and extracranial metastases, primary tumor histological type, EGFR mutation type, first-line treatment, therapy for BM, RT types, and interval between RT and diagnosis. Patients were categorized by age ( $<65$ years, $\geq 65$ years), sex (male, female), smoking status (never, slight, heavy), KPS $(\geq 70,<70)$, extracranial metastases (yes or no), number of BMs (1 tumor, 2 tumors, more than 2 tumors), primary tumor histology (adenocarcinoma, others), EGFR mutation type (exon 19 deletion, exon 21 L858R mutation), first-line treatment (chemotherapy, EGFR-TKIs), chemotherapy (single agent, dual agents), and RT types (WBRT, SRS, or combination). Finally, a graded prognostic assessment (GPA) was calculated for each patient to determine whether the cohorts shared similar prognostic features.

Subgroup analysis was performed based on first-line treatment regimens; patients were divided into 2 groups: chemotherapy $(n=28)$ and EGFR-TKIs $(n=17)$. Patients were also divided into 2 groups according to whether they received upfront RT (with an interval between RT and diagnosis of $\leq 3$ months), and it was found that 30 patients were treated with upfront RT and 15 received deferral RT instead. A major focus of the study was OS, which was defined as the duration of time from the start of therapy until death or the most recent follow-up.

\section{Statistical analysis}

Kaplan-Meier analysis was used to estimate OS, whereas log-rank testing was used to assess differences. OS was calculated from the date of BM diagnosis until the date of death. Finally, the Cox proportional hazard regression model (forward Wald method) was used for multivariate analysis of the groups to study the effect of prognostic factors from the
Kaplan-Meier single-variant test (mutation, GPA, number of intracranial lesions, extracranial metastasis) and clinical factors (first-line treatment, interval of BM) and to evaluate which factors were associated with patient survival as well as to analyze differences in the survival curves for each subgroup. All statistical analyses were performed using SPSS software, version 20.0 (IBM Corporation, Armonk, NY, USA).

\section{Results}

\section{Baseline characteristics of patients}

Twenty (44.4\%) of the 45 NSCLC patients diagnosed with BMs who were enrolled in the study were male. The median age of these patients was 56 years (ranging between 38 and 70 years), and 7 patients (15.6\%) had a KPS score of $\geq 90$. A total of 16 patients (35.6\%) were smokers, and 11 patients (23.9\%) had heavy smoking histories. These NSCLC cases included 41 adenocarcinoma and 4 non-adenocarcinoma cases. The EGFR exon 19 deletion mutation occurred in 22 (48.9\%) patients, and $23(51.1 \%)$ patients had the EGFR exon $21 \mathrm{~L} 858 \mathrm{R}$ mutation. Thirty-seven patients received icotinib (125 mg/3 times daily), 4 patients gefitinib $(250 \mathrm{mg} / \mathrm{d})$, 3 osimertinib ( $80 \mathrm{mg} / \mathrm{d})$, and 1 erlotinib $(150 \mathrm{mg} / \mathrm{d})$. While $14(31.1 \%)$ patients had a single intracranial lesion, $5(11.1 \%)$ patients had 2 lesions, 26 (57.8\%) had more than 2 lesions, and $27(60.0 \%)$ had extracranial metastasis. In total, 30 $(66.7 \%)$ patients received upfront RT. A total of 28 patients $(62.2 \%)$ were treated with pemetrexed-based chemotherapy as the first-line treatment, while $17(37.8 \%)$ received first-line EGFR-TKIs instead. Thirty-eight patients received WBRT (30Gy/10f/2W), 5 received SRS, and 1 received the combined SRS and WBRT. Twenty-six (57.8\%) patients had a GPA score between 0 and 2.0, and $19(42.2 \%)$ patients had a score between 2.5 and 4.0 . The patient characteristics at baseline are detailed in Table 1.

\section{Survival outcomes}

Survival analysis showed that the median OS from diagnosis of BM was 28 months for the whole cohort $(95 \% \mathrm{CI}$, 17.3-38.7 months) in Figure 1. Patients who were younger than 65 years had a significantly longer OS (32.8 months; 95\% CI, 23.7-41.9) than that of elderly patients (15.6 months; 95\% CI, 8.1-23.1; $P=0.001)$. Patients with the exon 19 deletion showed a trend toward a longer median OS (not reached) than that of patients with the exon 21 L858R mutation (23.2 months; 95\% CI, 0.22-1.13; $P=0.0969$ ) in Figure 2A. There was no difference in OS between the upfront RT group and the deferral group ( 28.0 vs 26.5 months, $P=0.74$ ) 


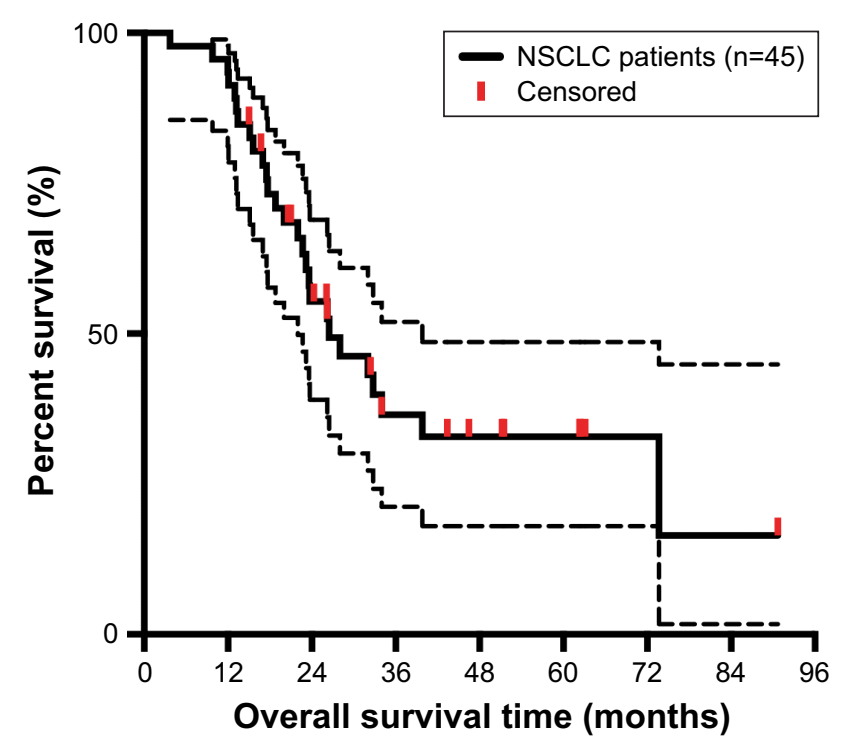

Figure I Kaplan-Meier curve illustrating OS of all NSCLC patients. Abbreviations: NSCLC, non-small-cell lung cancer; OS, overall survival.
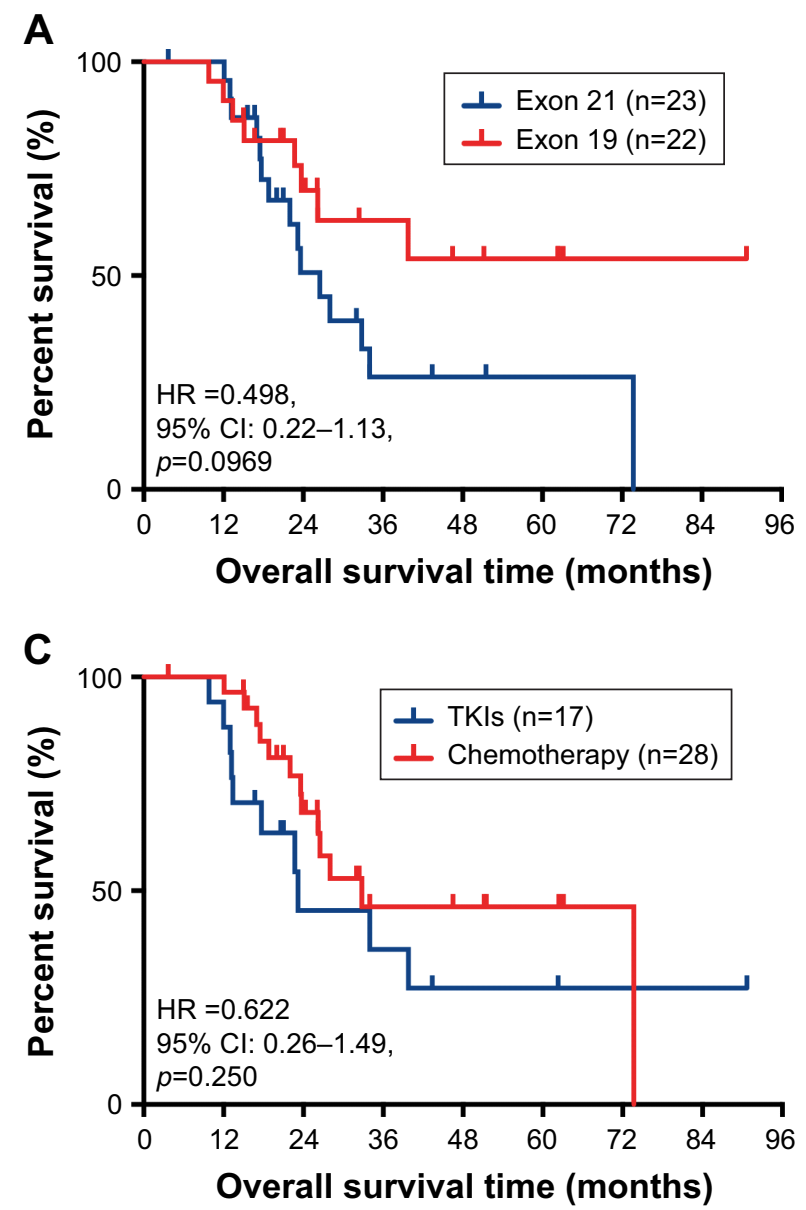

in Figure 2B. Similar results were found between the firstline chemotherapy group and the EGFR-TKI group (28.0 vs 23.2 months, $P=0.25$ ) in Figure $2 \mathrm{C}$. And we also found no statistical significance between the GPA score of 0-2 group and 2.5-4 group $(P=0.69)$ in Figure 2D.

Furthermore, the median intercranial progression-free survival (iPFS) rate of the whole cohort was 17.7 months (95\% CI, 12.4-23.0 months). However, the iPFS of 8 patients could not be assessed because the intercranial lesions were not evaluable. The first-line chemotherapy group showed an iPFS of 17.78 months, and the EGFR-TKI group showed and iPFS rate of 13.00 months; however, there was no statistically significant difference $(P=0.489)$. In the chemotherapy group, 25 patients were evaluable, and 15 received upfront RT.

As for intracranial lesions, 9 (19.6\%) patients had CR, 21 (45.7\%) had PR, 15 (32.6\%) had SD, and 1 (2.2\%)
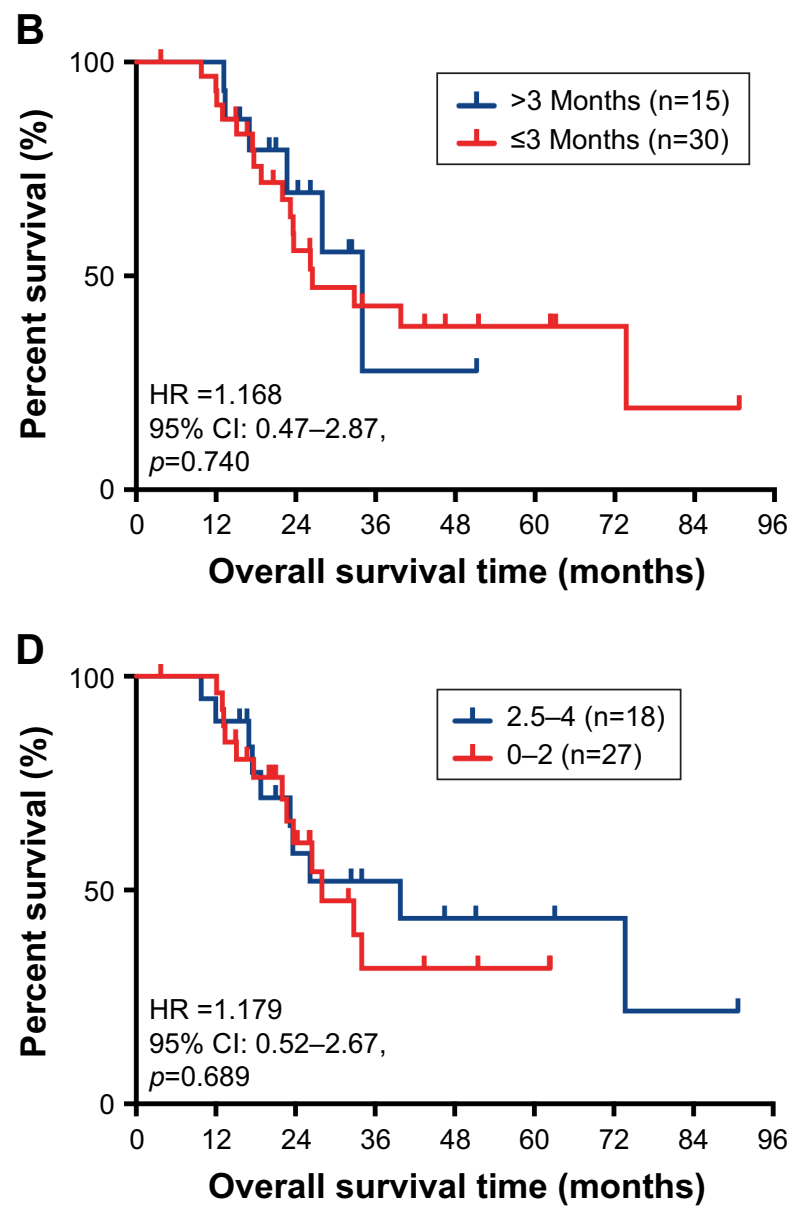

Figure 2 Kaplan-Meier curve analyses of patient OS after EGFR-TKI, chemotherapy, and WBRT.

Notes: (A) Kaplan-Meier curve was stratified by EGFR mutation types (exon 19 deletion vs exon 2I L858R mutation). (B) Kaplan-Meier curves were stratified by interval time of RT. (C) Kaplan-Meier curves were stratified by different first-line treatments (chemotherapy vs EGFR-TKIs). (D) Kaplan-Meier survival curve illustrating OS based on GPA scores.

Abbreviations: GPA, graded prognostic assessment; OS, overall survival; EGFR-TKI, epidermal growth factor receptor-tyrosine kinase inhibitor; WBRT, whole brain radiation therapy; HR, hazard ratio; $\mathrm{RT}$, radiation therapies. 
Table 2 Multivariate Cox regression analyses of iPFS and OS

\begin{tabular}{|c|c|c|c|c|c|c|}
\hline \multirow[t]{2}{*}{ Variable } & \multicolumn{3}{|l|}{ iPFS } & \multicolumn{3}{|l|}{ OS } \\
\hline & $P$-value & HR & $95 \% \mathrm{Cl}$ & $P$-value & HR & $95 \% \mathrm{Cl}$ \\
\hline Mutation & 0.327 & 0.538 & $0.156-1.855$ & 0.017 & 3.272 & $1.24-8.62$ \\
\hline RTOG GPA grade & 0.676 & $\mathrm{I} .34 \mathrm{I}$ & $0.339-5.306$ & 0.206 & 2.174 & $0.65-7.24$ \\
\hline Number of intracranial metastases & 0.192 & 0.686 & $0.390-1.208$ & 0.229 & 0.725 & $0.42-1.22$ \\
\hline Extracranial metastasis & 0.919 & 0.934 & $0.252-3.464$ & 0.070 & 2.858 & $0.91-8.90$ \\
\hline First-line treatment & 0.296 & 1.964 & $0.553-6.966$ & 0.114 & 2.124 & $0.83-5.39$ \\
\hline Interval of BM & 0.309 & 1.945 & $0.540-7.004$ & 0.482 & 1.407 & $0.54-3.64$ \\
\hline
\end{tabular}

Note: Variables with $P<0.05$ in univariate analysis were included in the multivariate analysis.

Abbreviations: BM, brain metastasis; GPA, graded prognosis assessment; HR, hazard ratio; iPFS, intracranial progression-free survival; RTOG, radiation therapy oncology group; OS, overall survival.

had PD in the EGFR-TKI group. The objective response rate was $65.2 \%$. In the chemotherapy group, although the intracranial response could not be evaluated in 7 patients, 7 (19.4\%) patients had CR, 20 (55.6\%) had PR, 10 (27.8\%) had SD, and $1(2.8 \%)$ had PD. The objective response rate was $75.0 \%$. Patients who were younger than 65 years had a significantly longer OS (32.8 months; 95\% CI, 23.7-41.9) than that of elderly patients (15.6 months; $95 \% \mathrm{CI}, 8.1-23.1$; $P=0.001$ ).

In the multivariate analysis, the prognosis independently correlated with the EGFR mutation type (adjusted hazard ratio, 3.27; 95\% CI, 1.24-8.62; $P=0.017$ ). Notably, gender, age, smoking history, KPS, number of metastatic lesions in the brain, absence of extracranial disease, first-line treatment, and RT types were not found to significantly influence the prognosis. Although patients with extracranial metastases showed the strongest trend toward decreased OS, it was not statistically significant $(P=0.070)$. Patients with a higher GPA score had a better survival rate than that of patients with a lower score; however, this also was not statistically significant $(P=0.206)$ (Table 2$)$.

\section{Discussion}

To date, the treatment of advanced NSCLC patients with BM remains a significant clinical challenge. Therapeutic modalities for controlling BM include WBRT, SRS, surgery, and chemotherapy, but the efficacy of these treatments remains poor. The use of first-line EGFR-TKI therapy for patients with metastatic EGFR-mutant lung adenocarcinoma is based upon a randomized trial demonstrating improved PFS compared to standard chemotherapy. ${ }^{19}$

For patients with mutations like the exon 19 deletion and the exon 21 L858R mutation, EGFR-TKIs are recommended as a first-line treatment. In our study, although we found that patients with the exon 19 deletion had a longer median OS than patients with the exon 21 L858R mutation (not reached vs 23.2 months), this difference was not statistically significant $(P=0.031)$. This result suggested that these 2 mutations are different to some extent. Recent studies also reported that patients with the exon 19 deletion and the exon 21 L858R mutation have different survival outcomes in response to EGFR-TKIs and chemotherapy. ${ }^{20-22}$ EGFR-TKI treatment was demonstrated to be more effective in patients with the exon 19 deletion than in patients with the exon 21 L858R mutation. ${ }^{20,21}$ In contrast, in a metaanalysis by Lee et al, ${ }^{20}$ patients who were not treated with EGFR-TKIs were randomly assigned to chemotherapy, and patients with the exon 21 L858R mutation had a statistically significantly longer PFS than those with the exon 19 deletion. These findings suggest that the difference in the prognosis between patients with the exon 19 deletion and the exon 21 L858R mutation might be related to the efficacy of the EGFR-TKIs. ${ }^{23}$

There was no improvement in the OS of patients who received upfront RT compared with deferral RT group in our study ( 28.0 vs 26.5 months, $P=0.74$ ), which varies from many previous studies. A systematic review and metaanalysis of 12 studies found that, compared with upfront erlotinib alone, upfront cranial RT (SRS or WBRT) followed by erlotinib improved the OS and intracranial PFS in patients with EGFR-mutant NSCLC who developed BM. ${ }^{17}$ Another multi-institutional analysis by Magnuson et $\mathrm{al}^{6}$ also demonstrated that the use of upfront EGFR-TKI and deferral of RT associated with inferior OS in patients with EGFR-mutant NSCLC who developed BM. In addition, SRS followed by EGFR-TKI associated with the longest OS and allowed patients to avoid the potential neurocognitive sequelae of WBRT. ${ }^{24}$ However, a recent Phase III trial (BRAIN) showed that icotinib was associated with a significantly longer intracranial PFS than that of whole-brain irradiation 
plus chemotherapy, indicating that icotinib might be a better first-line therapeutic option for this patient population. We assume that our conclusion may be due to factors such as small sample size and the nonrandomized nature of the study. However, further randomized controlled trials are needed to examine the correlation between the timing of RT interval and OS and to define the optimal timing of RT.

In our study, due to the limitation of the period that patients were treated and the limitation of the medical insurance policy, more than half of the patients received chemotherapy as first-line treatment. We compared the OS of the first-line chemotherapy group and the first-line EGFR-TKI group and found no statistically significant difference $(P=0.50)$. The OS of the chemotherapy group was somewhat better than the EGFR-TKI group (28.0 vs 23.2 months). Barlesi et $\mathrm{al}^{8}$ previously demonstrated that pemetrexed-based chemotherapy has a great activity as well as a good safety profile in managing the NSCLC patients with inoperable BM. Thus, the patients who we enrolled were limited to those who had received pemetrexed treatment. Ten randomized controlled trials showed that there was no significant difference in the OS between first-line TKI treatment and first-line chemotherapy. For iPFS, the first-line chemotherapy group showed a longer iPFS than that of the EGFR-TKI group (17.78 vs 13.00 months, $P=0.590$ ). This result is possibly because most patients of the chemotherapy group received upfront RT in our study, which could efficiently prolong the PFS. And the EGFR-TKI group may show clinical resistance after the use of the first-generation EGFR-TKIs. The responses of first-generation EGFR-TKIs typically last for 6-12 months before resistance develops, which is coincide with our results. ${ }^{15}$

There are many limitations in the current study. First, we used a retrospective design, and due to the variety of exclusion factors, there may have been bias in choosing patients for enrollment. Therefore, the results reported here are not entirely representative of a large sample population. Second, the sample size of this study was quite small, which may have affected its statistical power. Third, based on the baseline clinical characteristics of the patients, the treatment groups were not homogeneous. Fourth, the choice for treatment was not random because it was determined by the willingness of both the physicians and the patients, which may have led to deviations between the 2 treatment groups. Fifth, patients enrolled in our study were not tested for the EGFR status after the progression of EGFR-TKIs, and so the acquired mutations were unknown and the next generation of EGFRTKIs was not applied. Finally, follow-up data on toxicities, cognitive impairment, and quality of life were lacking, and we were therefore unable to analyze these factors.

\section{Conclusion}

In summary, our results suggest NSCLC patients with BMs benefited from the combination and sequential therapies of EGFR-TKIs, chemotherapy and RT (WBRT or SRS). In addition, patients with the EGFR exon 19 deletion may have a better OS than patients with the exon 21 L858R mutation. However, the optimal timing of RT interval remains to be explored. And the ideal sequence about the application of third- or even fourth-generation EGFR-TKIs is unclear. Furthermore, immunotherapy provides a new treatment option. Larger prospective randomized clinical trials are urgently needed to explore the optimal treatment combination for BM in patients with EGFR-mutant NSCLC.

\section{Disclosure}

The authors report no conflicts of interest in this work.

\section{References}

1. Gallego Perez-Larraya J, Hildebrand J. Brain metastases. Handb Clin Neurol. 2014;121:1143-1157.

2. Johung KL, Yeh N, Desai NB, et al. Extended survival and prognostic factors for patients with ALK-rearranged non-small-cell lung cancer and brain metastasis. J Clin Oncol. 2016;34(2):123-129.

3. Shin DY, Na II, Kim CH, Park S, Baek H, Yang SH. EGFR mutation and brain metastasis in pulmonary adenocarcinomas. J Thorac Oncol. 2014;9(2):195-199.

4. Sperduto PW, Kased N, Roberge D, et al. Summary report on the graded prognostic assessment: an accurate and facile diagnosis-specific tool to estimate survival for patients with brain metastases. J Clin Oncol. 2012;30(4):419-425.

5. Gerber NK, Yamada Y, Rimner A, et al. Erlotinib versus radiation therapy for brain metastases in patients with EGFR-mutant lung adenocarcinoma. Int J Radiat Oncol Biol Phys. 2014;89(2):322-329.

6. Magnuson WJ, Yeung JT, Guillod PD, Gettinger SN, Yu JB, Chiang VL. Impact of deferring radiation therapy in patients with epidermal growth factor receptor-mutant non-small cell lung cancer who develop brain metastases. Int J Radiat Oncol Biol Phys. 2016;95(2):673-679.

7. Yang TJ, Wu AJ. Cranial irradiation in patients with EGFR-mutant non-small cell lung cancer brain metastases. Transl Lung Cancer Res. 2016;5(1):134-137.

8. Barlesi F, Gervais R, Lena H, et al. Pemetrexed and cisplatin as firstline chemotherapy for advanced non-small-cell lung cancer (NSCLC) with asymptomatic inoperable brain metastases: a multicenter phase II trial (GFPC 07-01). Ann Oncol. 2011;22(11):2466-2470.

9. Bailon O, Chouahnia K, Augier A, et al. Upfront association of carboplatin plus pemetrexed in patients with brain metastases of lung adenocarcinoma. Neuro Oncol. 2012;14(4):491-495.

10. Park SJ, Kim HT, Lee DH, et al. Efficacy of epidermal growth factor receptor tyrosine kinase inhibitors for brain metastasis in non-small cell lung cancer patients harboring either exon 19 or 21 mutation. Lung Cancer. 2012;77(3):556-560.

11. Cai L, Zhu JF, Zhang XW, et al. A comparative analysis of EGFR mutation status in association with the efficacy of TKI in combination with WBRT/SRS/surgery plus chemotherapy in brain metastasis from non-small cell lung cancer. J Neurooncol. 2014;120(2):423-430. 
12. Kim JE, Lee DH, Choi Y, et al. Epidermal growth factor receptor tyrosine kinase inhibitors as a first-line therapy for never-smokers with adenocarcinoma of the lung having asymptomatic synchronous brain metastasis. Lung Cancer. 2009;65(3):351-354.

13. Wang S, Cang S, Liu D. Third-generation inhibitors targeting EGFR T790M mutation in advanced non-small cell lung cancer. $J$ Hematol Oncol. 2016;9:34.

14. Wang S, Tsui ST, Liu C, Song Y, Liu D. EGFR C797S mutation mediates resistance to third-generation inhibitors in T790M-positive non-small cell lung cancer. J Hematol Oncol. 2016;9(1):59.

15. Wang S, Song Y, Yan F, Liu D. Mechanisms of resistance to thirdgeneration EGFR tyrosine kinase inhibitors. Front Med. 2016;10(4): 383-388.

16. $\mathrm{Su} \mathrm{S}, \mathrm{Wu}$ YL. Clinical trials of tyrosine kinase inhibitors for lung cancer in China: a review. J Hematol Oncol. 2017;10(1):147.

17. Soon YY, Leong CN, Koh WY, Tham IW. EGFR tyrosine kinase inhibitors versus cranial radiation therapy for EGFR mutant non-small cell lung cancer with brain metastases: a systematic review and metaanalysis. Radiother Oncol. 2015;114(2):167-172.

18. Jiang T, Min W, Li Y, Yue Z, Wu C, Zhou C. Radiotherapy plus EGFR TKIs in non-small cell lung cancer patients with brain metastases: an update meta-analysis. Cancer Med. 2016;5(6):1055-1065.

19. Rosell R, Carcereny E, Gervais R, et al. Erlotinib versus standard chemotherapy as first-line treatment for European patients with advanced EGFR mutation-positive non-small-cell lung cancer (EURTAC): a multicentre, open-label, randomised phase 3 trial. Lancet Oncol. 2012;13(3):239-246.
20. Lee CK, Wu YL, Ding PN, et al. Impact of specific epidermal growth factor receptor (EGFR) mutations and clinical characteristics on outcomes after treatment with EGFR tyrosine kinase inhibitors versus chemotherapy in EGFR-mutant lung cancer: a meta-analysis. J Clin Oncol. 2015;33(17):1958-1965.

21. Yang JC, Wu YL, Schuler M, et al. Afatinib versus cisplatin-based chemotherapy for EGFR mutation-positive lung adenocarcinoma (LUXLung 3 and LUX-Lung 6): analysis of overall survival data from two randomised, phase 3 trials. Lancet Oncol. 2015;16(2):141-151.

22. Shigematsu H, Lin L, Takahashi T, et al. Clinical and biological features associated with epidermal growth factor receptor gene mutations in lung cancers. J Natl Cancer Inst. 2005;97(5):339-346.

23. Ke EE, Zhou Q, Zhang QY, et al. A higher proportion of the EGFR T790M mutation may contribute to the better survival of patients with exon 19 deletions compared with those with L858R. J Thorac Oncol. 2017;12(9):1368-1375.

24. Chen $\mathrm{W}, \mathrm{Xu} \mathrm{XK}, \mathrm{Li} \mathrm{JL}$, et al. MALAT1 is a prognostic factor in glioblastoma multiforme and induces chemoresistance to temozolomide through suppressing miR-203 and promoting thymidylate synthase expression. Oncotarget. 2017;8(14):22783-22799.
OncoTargets and Therapy

\section{Publish your work in this journal}

OncoTargets and Therapy is an international, peer-reviewed, open access journal focusing on the pathological basis of all cancers, potential targets for therapy and treatment protocols employed to improve the management of cancer patients. The journal also focuses on the impact of management programs and new therapeutic agents and protocols on

\section{Dovepress}

patient perspectives such as quality of life, adherence and satisfaction. The manuscript management system is completely online and includes a very quick and fair peer-review system, which is all easy to use. Visit http://www.dovepress.com/testimonials.php to read real quotes from published authors. 